\title{
МОДЕЛЬ И МЕТОД СЖИГАНИЯ В ТЕПЛОЭНЕРГЕТИЧЕСКОЙ УСТАНОВКЕ УГЛЕВОДОРОДНОГО ГАЗА ПЕРЕМЕННОГО СОСТАВА
}

\author{
Лысюк А. В. ${ }^{1}$, Бондаренко А. В. ${ }^{2}$, Максимов М. М. ${ }^{3}$, Брунеткин А. И. ${ }^{4}$ \\ 1,3,4 Одесский национальный политехнический университет, Украина \\ ${ }^{2}$ Национальный университет «Одесская морская академия», Украина \\ E-mail: lysyuk92@gmail.com ${ }^{1}$, bondandrey@ukr.net ${ }^{2}$,prof.maksimov@gmail.com ${ }^{3}$, alexbrun@ rambler.ru ${ }^{4}$ \\ Copyright (C) 2014 by author and the journal -Automation technological and business - processesll. \\ This work is licensed under the Creative Commons Attribution International License (CC BY). \\ http://creativecommons.org/licenses/by/4.0/
}

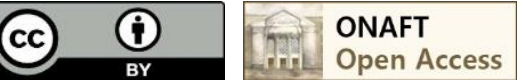

Аннотация: Определение условной формульл газообразного углеводородного топлива основано на модельных представлениях в соответствии с законами сохранения вещества, Дальтона, химического равновесия по парциальным давлениям. Определение динамических характеристик теплоэнергетического оборудования основано на уравнениях материального и теплового балансов, законов конвективного и лучистого теплообмена и свойств сжимаемой газообразной среды. Предложен метод обеспечения заданной тепловой нагрузки теплогенерирующей установки основаный на вычислении условной формулы газообразного углеводородного топлива и определении динамических характеристик теплогенерирующей установки.

Keywords: Углеводородный газ, модель сжигания, метод сжигания несертифицированного топлива, теплоэнергетическая установка, максимальная температура горения.

Текущие значения удельных энергетических показателей совокупного национального дохода Украины в разы превышает аналогичные показатели стран Евросоюза. Причина определяется не только технологической отсталостью национальной экономики, но и отсутствием в ней конкуренции на энергетическом рынке. Использование вторичных энергоресурсов нефтепереработки, металлургии биотехнологий, переработки твердых бытовых отходов, качественный состав которых меняется во времени, делает невозможным их сжигание с регламентированным поддержанием заданных энергетических характеристик. Обеспечение энергетических установок несертифицированным углеводородным газом для снижения энергоемкости совокупного национального продукта Украины стратегически целесообразно для разработки и внедрения новых методов и моделей, которые позволят за счет вторичного использования таких углеводородных газов повысить эффективность технологических процессов сжигания топлива [1].

Разработки моделей и методов процессов сжигания углеводородного газа переменного состава для обеспечения заданной тепловой нагрузки энергетической установки и определяет актуальность данной статьи.

1. Анализ литературных данных и постановка проблеми

В работах [2,3] изучены этапы горения углеводородного газа в воздухе. В основу модели был положен кинетический механизм. Получены аналитические выражения энтальпии горения и характерной температуры. Для обедненного топлива численные и натурные эксперименты совпали. Результаты по скоростям горения получились завышенными для стехиометрического и богатого пламени.

Показано, что на первом этапе выделение энергии уменьшается, а задержка воспламенения увеличивается нелинейно с повышением октанового числа и уменьшением начального давления. Скорость выделения энергии определяется, прежде всего, скоростью окисления СО. Предварительный анализ [4,6] построения модели показал, что для создания модели окисления смеси газов необходимо использовать подход, состоящий подход, основанный на моделировании цепного механизма. В открытой литературе не обнаружено никаких аналитических методов и моделей позволяющих поддерживать заданные энергетические характеристики энергетического оборудования при изменение свойств углеводородного топлива.

2. Цель и задачи исследования

Целью статьи является разработка модели и метода сжигания углеводородного газа переменного состава за счет 
изменения отношения топливо/воздух для получения максимальной температуры сгорания топлива в теплоэнергетической установке.

Задачи, решаемые в данной статье. Разработка математической модели сжигания углеводородных газов переменного состава в камере сгорания теплоэнергетической установки. Разработка метода обеспечения оптимального постоянства топливо/воздух для получения максимальной температуры сгорания топлива.

3. Построение модели сжигания углеводородного газа переменного состава.

Для решения задачи использования теплогенерирующими установками несертифицированных углеводородных газов необходимо разработать математическую модель и метод определения расхода продуктов сгорания. Принято окислитель - кислород, горючее - углеводородный газ. Для определения состава будем использовать измерительные датчики, которыми оснащается теплогенерирующая установка [2]. Это расходомеры окислителя и горючего и измерительный прибор температуры пламени [3]. В состав углеводородного газа входят элементы «С» - углерод и «Н» - водород, а его конкретный состав и коэффициент « $\alpha$ » (коэффициент избытка окислителя) неизвестны. Горючее и окислитель находятся в газообразном состоянии. Исходные данные: $\dot{V}_{o \kappa} ; \quad \dot{V}_{\text {гор }} ;{ }^{(1)} T ; \quad \dot{V}_{o \kappa} ; \quad \dot{V}_{\text {гор }} ;$ ${ }^{(2)} T ; \quad \mu_{C} ; \mu_{H} ; \mu_{O} ; P_{\Sigma} ; \delta$, где $\dot{V}_{o \kappa}-$ объёмный расход окислителя; $\dot{V}_{\text {гор }}$ - объёмный расход горючего; $T-$ температура в камере при данных расходах горючего и окислителя; ${ }^{(1)} X ;{ }^{(2)} X-$ верхний индекс, указывающий на замер параметров при соответствующих расходах $\dot{V}_{\text {гор }} \cdot \dot{V}_{\text {ок }} ; \mu_{C}, \mu_{H}, \mu_{O}$ - соответствующие массы углерода, водорода, кислорода; $P_{\Sigma}$ - давление в камере; $\delta$ - коэффициент нижней релаксации необходим для численных расчетов.

Предварительно вычисляются:

$$
{ }^{(1)} a_{\Gamma}={ }^{(1)} \dot{V}_{\Gamma} /{ }^{(1)} \dot{V}_{o \kappa}+{ }^{(1)} \dot{V}_{\Gamma} ;{ }^{(2)} a_{\Gamma}={ }^{(2)} \dot{V}_{\Gamma} /{ }^{(2)} \dot{V}_{o \kappa}+{ }^{(2)} \dot{V}_{\Gamma} ;{ }^{(1)} a_{o \kappa}={ }^{(1)} \dot{V}_{o \kappa} /{ }^{(1)} \dot{V}_{o \kappa}+{ }^{(1)} \dot{V}_{\Gamma} ;{ }^{(2)} a_{o \kappa}={ }^{(2)} \dot{V}_{o \kappa} /{ }^{(2)} \dot{V}_{o \kappa}+{ }^{(2)} \dot{V}_{\Gamma} ; \mu \mu_{C O} ;
$$
$\mu_{\mathrm{CO}_{2}} ; \mu_{\mathrm{H}_{2}} ; \mu_{\mathrm{O}_{2}} ; \mu_{\mathrm{H}_{2} \mathrm{O}} ; \mu_{\mathrm{OH}}$.

При заданных температуре и давлении рассмотрим вычисление парциальных давлений продуктов сгорания углеводородов в кислороде. Из числа возможных индивидуальных веществ продуктов сгорания рассмотрим основные $\mathrm{CO}, \mathrm{CO}_{2}, \mathrm{H}_{2} \mathrm{O}, \mathrm{OH}, \mathrm{H}_{2}, \mathrm{O}_{2}, \mathrm{H}, \mathrm{O}, \mathrm{C}$.

Молекулярная масса условной формулы топлива определяется в виде

$$
\mu_{m}=\mu_{\Gamma}+\dot{V_{o \kappa}} / \dot{V}_{\Gamma} \cdot \mu_{o \kappa}, \quad \mu_{m}={s_{H}}^{(\Gamma)} \cdot \mu_{H}+{\theta_{C}}^{(\Gamma)} \cdot \mu_{C}+\dot{V_{o \kappa}} / \dot{V}_{\Gamma} \cdot a \cdot \mu_{O},
$$

где $\dot{V_{o \kappa}}, \dot{V_{\Gamma}}$ - объёмные расходы окислителя и горючего соответственно; $\mu_{о \kappa}, \mu_{\Gamma}-$ молекулярные массы окислителя и горючего соответственно; $\mu_{m}$ - молекулярная масса условной формулы топлива.

Для окислителя $\mu_{о \kappa}=2 \cdot \mu_{O}{ }^{(о \kappa)}=a \cdot \mu_{O}{ }^{(о к)}$,

где $\mu_{O}=15,9994$ - атомная масса кислорода; $a=2$ - количество атомов кислорода в окислителе.

Для горючего $\mu_{\Gamma}={\theta_{H}}^{(\Gamma)} \cdot \mu_{H}+{\theta_{C}}^{(\Gamma)} \cdot \mu_{C}$,

где $\mu_{H}=1,00795$ - атомная масса водорода; $\mu_{C}=12,0108$ - атомная масса углерода; $6_{H}{ }^{(\Gamma)},{ }_{C}{ }^{(\Gamma)}-$ количество атомов водорода и углерода в условной формуле горючего.

Если горючее состоит из одного вещества, то и соответствуют количеству соответствующих атомов в химической

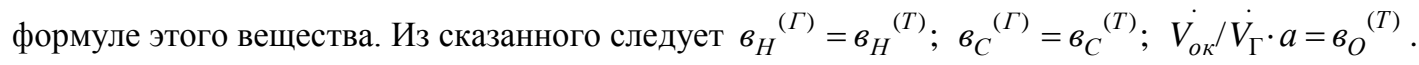

Здесь $B_{H}{ }^{(T)},{ }^{B_{C}}{ }^{(T)},{ }{ }^{(T)}$ - количество атомов водорода, углерода и кислорода в условной формуле топлива. Величины $b_{H}{ }^{(\Gamma)}$ и ${ }_{{ }_{C}}{ }^{(\Gamma)}-$ неизвестные и подлежат определению. Тогда набор уравнений для любой точки замера объемного расхода запишется в виде шести уравнений закона действующих масс.

$P_{C} \cdot P_{O} / P_{C O}=\kappa_{1}(T), \quad P_{C} \cdot P_{O}{ }^{2} / P_{C O_{2}}=\kappa_{2}(T), \quad P_{H}{ }^{2} \cdot P_{O} / P_{H_{2} O}=\kappa_{3}(T), \quad P_{H} \cdot P_{O} / P_{O H}=\kappa_{4}(T), \quad P_{H}{ }^{2} / P_{H_{2}}=\kappa_{5}(T)$, $P_{O}^{2} / P_{O_{2}}=\kappa_{6}(T)$.

Предварительно рассчитывались реакции диссоциации между рассматриваемыми веществом и составными элементами.

Уравнения сохранения вещества

$M_{T} \cdot{ }{ }_{C}^{(T)}={ }^{(\Gamma \Gamma)} \cdot M_{T}=P_{C O}+P_{C O_{2}}+P_{C}$, 


\section{http://atbp.onaft.edu.ua/}

$M_{T} \cdot{ }^{(T)}={ }^{(T)}{ }^{(Г \Gamma)} \cdot M_{T}=2 \cdot P_{H_{2} O}+2 \cdot P_{H_{2}}+P_{O H}+\mathrm{P}_{H}$,

$M_{T} \cdot{ }{ }^{(T)}=\dot{V_{o K}} / \dot{V}_{\Gamma} \cdot a \cdot M_{T}=P_{C O}+2 \cdot P_{C_{2}}+P_{H_{2} O}+\mathrm{P}_{O H}+2 \cdot P_{O_{2}}+\mathrm{P}_{O}$,

Закон Дальтона $P_{C O}+P_{C_{2}}+P_{H_{2}}+P_{O_{2}}+\mathrm{P}_{H_{2} O}+P_{O H}+\mathrm{P}_{H}+\mathrm{P}_{O}+\mathrm{P}_{C}=\mathrm{P}_{\Sigma}$,

Замыкающие уравнение $\left(\mathrm{I}_{(\Gamma)}+\dot{V}_{o \kappa} / \dot{V}_{\Gamma} \cdot I_{(O)}\right) \cdot \mathrm{M}_{T}=\sum_{q} P_{q} \cdot \mathrm{I}_{q}$

Уравнения записаны для одного из измерений, например ${ }^{(1)} V_{o \kappa},{ }^{(1)} V_{\Gamma},{ }^{(1)} T$.

При этом, в качестве неизвестных будут выступать ${ }^{(1)} P_{i},{ }^{(1)} M_{T},{ }^{(1)} B_{H}{ }^{(\Gamma)},{ }^{(1)}{ }_{C}{ }_{C}^{(\Gamma)}$. Для второго замера ${ }^{(2)} V_{o \kappa},{ }^{(2)} V_{\Gamma},{ }^{(2)} T$ может быть записана вторая часть уравнений в аналогичном виде. В этом случае, как неизвестные будут выступать ${ }^{(2)} P_{i},{ }^{(2)} M_{T},{ }^{(2)} \boldsymbol{B}_{H}{ }^{(\Gamma)},{ }^{(2)} \boldsymbol{B}_{C}{ }^{(\Gamma)}$.

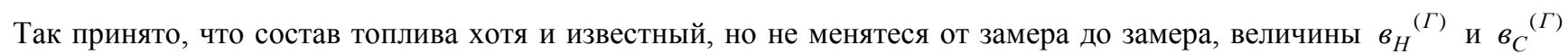
однаковые для первой (1-ый замер) и второй (2-ый замер) частей общей системы уравнений.

Для повышения устойчивости решения необходимо найти жесткую связь между определяемыми величинами, которые не изменяются в процессе вычислений состава продуктов сгорания. С этой целью была определена связь между количеством атомов $[C]$ и $[H]$ в горючем при $\alpha=1$ (стехиометрическом соотношении компонентов). $\dot{V}_{\text {ок }} / \dot{V}_{\Gamma}=\alpha \cdot \chi_{0}=\chi_{0}$.

С другой стороны, при стехиометрическом соотношении количество максимальных валентностей окислительных элементов равно количеству максимальных валентностей восстановительных элементов. Поэтому применительно к углеводородам и кислорода можно записать такой баланс: $C_{b C} H_{b H}+\dot{V}_{o \kappa} / \dot{V}_{\Gamma} \cdot O_{2}=b_{C} \cdot C O_{2}+b_{H} / 2 \cdot H_{2} O$, а из него баланс по атомам кислорода: $2 \cdot \dot{V}_{o \kappa} / \dot{V}_{\Gamma}=2 \cdot b_{C}+b_{H} / 2$,

Уравнения являются основой рассматриваемой модели и также являются нелинейными. Решение их может быть получено по методу Ньютона путем линеаризации исходных уравнений и решение системы линейных уравнений относительно поправок искомых величин. Искомые величины определяются в итерационном процессе путем составления начальных приближений с найденными поправками [4].

Для проверки работоспособности предложенной модели и выявления особенностей, возникающих в процессе вычислений, была использована топливная пара $\mathrm{CH}_{1,956}$ (керосин) $+\mathrm{O}_{2}$ (жидкий кислород) [5]. Для решения задачи в тестовом варианте взяты точки, соответствующие $\alpha=0.4 ; 0.7 ; 1.0$. Проверочный расчет выполнялся для двух отношений объемного расхода $\dot{V}^{(1)} o / \dot{V}^{(1)} \Gamma=0.5956$ и $\dot{V}^{(2)} o / \dot{V}^{(2)} \Gamma=1.0423$, соответствующих $\alpha^{(1)}=0.4$ и $\alpha^{(2)}=0.7$, а также температурам $T^{(1)}=2125 K$ и $T^{(1)}=3081 K$. Отношение объемного расхода при стехиометрическом соотношении принято $\dot{V}_{O}{ }^{(c m e x)} / \dot{V}_{\Gamma}{ }^{(c m e x)}=1.489$ см. табл 1 .

Анализ результатов расчетов показывает, что решение получено с высокой степенью математической точности, то есть модель не дает каких-то искажений, связанных с допущениями или неточностей численных расчетов. Незначительные расхождения в результатах (знаковые) могут быть объяснены тем, что эталонные данные были получены с такой же точностью. Быстрая сходимость результатов расчетов 50 итераций позволяет, хотя и косвенным образом, судить о высокой устойчивости численного решения.

Таблица 1 - Результаты расчета по расширенной модели

\begin{tabular}{|c|c|c|c|}
\hline $\begin{array}{c}\text { Состав } \\
\text { продуктов } \\
\text { сгорания }\end{array}$ & $\begin{array}{c}\text { Нач. } \\
\text { прибл }\end{array}$ & $\begin{array}{c}\text { Точн. реш. } \\
\text { (прям.задача) }\end{array}$ & $\begin{array}{c}\text { Данные } \\
\text { по работе [5] }\end{array}$ \\
\hline 1 & 2 & 3 & 4 \\
\hline $\mathrm{P}^{(1)}$ СО & 1,0 & 0,4869 & 0,4432 \\
\hline $\mathrm{P}^{(1)} \mathrm{CO} 2$ & 1,0 & 0,0181 & 0,0203 \\
\hline $\mathrm{P}^{(1)}{ }_{22 O}$ & 1,0 & 0,0784 & 0,1011 \\
\hline $\mathrm{P}^{(1)} \mathrm{OH}$ & 0,1 & 0,0001 & 0,0001 \\
\hline
\end{tabular}




\begin{tabular}{|l|c|c|c|}
\hline \multicolumn{4}{|c}{ Продолжение таблицы 1 } \\
\hline 1 & 2 & 3 & 4 \\
\hline $\mathrm{P}^{(1)}{ }_{\mathrm{H} 2}$ & 0,1 & 0,4143 & 0,4332 \\
\hline $\mathrm{P}^{(1)}{ }_{\mathrm{O} 2}$ & 0,1 & 0,0000 & 0,0000 \\
\hline $\mathrm{P}^{(1)}{ }_{\mathrm{H}}$ & 0,1 & 0,0022 & 0,0023 \\
\hline $\mathrm{P}^{(1)}{ }_{\mathrm{O}}$ & 0,1 & 0,0000 & 0,0000 \\
\hline $\mathrm{P}^{(1)}{ }_{\mathrm{C}}$ & 0,1 & 0,0000 & 0,0000 \\
\hline $\mathrm{M}^{(1)}{ }_{\mathrm{T}}$ & 1,0 & 0,5050 & 0,4910 \\
\hline $\mathrm{P}^{(2)}{ }_{\mathrm{CO}}$ & 1,0 & 0,3440 & 0,3172 \\
\hline $\mathrm{P}^{(2)}{ }_{\mathrm{CO}}$ & 1,0 & 0,1131 & 0,1042 \\
\hline $\mathrm{P}^{(2)}{ }_{\mathrm{H} 2 \mathrm{O}}$ & 1,0 & 0,2761 & 0,3043 \\
\hline $\mathrm{P}^{(2)}{ }_{\mathrm{OH}}$ & 0,1 & 0,0592 & 0,0584 \\
\hline $\mathrm{P}^{(2)}{ }_{\mathrm{H} 2}$ & 0,1 & 0,1094 & 0,1213 \\
\hline $\mathrm{P}^{(2)}{ }_{\mathrm{O} 2}$ & 0,1 & 0,0130 & 0,0117 \\
\hline $\mathrm{P}^{(2)}{ }_{\mathrm{H}}$ & 0,1 & 0,0638 & 0,0641 \\
\hline $\mathrm{P}^{(2)}{ }_{\mathrm{O}}$ & 0,1 & 0,0213 & 0,0191 \\
\hline $\mathrm{P}^{(2)}{ }_{\mathrm{C}}$ & 0,1 & 0,0000 & 0,0000 \\
\hline $\mathrm{M}^{(2)}{ }_{\mathrm{T}}$ & 1,0 & 0,4571 & 0,4464 \\
\hline $\mathrm{b}_{\mathrm{H}}$ & 1,0 & 1,9560 & 2,1808 \\
\hline $\mathrm{b}_{\mathrm{C}}$ & 1,0 & 1,0000 & 0,9438 \\
\hline $\mathrm{I}_{\Gamma}{ }$ & -12000 & -27238 & -30527 \\
\hline
\end{tabular}

Положительные результаты проверки адекватности модели показали, что предложенная математическая модель вычисления условной формулы газообразного углеводородного топлива, основанная на законах сохранения вещества, Дальтона, химического равновесия по парциальным давлениям позволяет определить количественный состав его условной формулы, энтальпию (теплотворную способность) и состав продуктов сгорания.

4. Математическая модель теплогенерирующей установки.

Ниже представлена модель обеспечения постоянства заданных характеристик генерации теплоты при изменении во времени качественного состава углеводородного газа [6]. Уравнение динамики теплового баланса для лучистого теплообмена:

$$
\left(Q_{p}^{H} \cdot G_{\text {газа }}\right)-G_{R} \cdot C_{R} \cdot T_{R}=k_{1}\left(T_{\text {in }}^{4}-T_{\text {оut }}^{4}\right)+m_{R} \cdot C_{R} \frac{d T_{R}}{d \tau},
$$

где $Q_{p}{ }_{p}$ - теплотворная способность, Вт; $G_{\text {газа }}$ - расход углеводородного газа, кг/с; $G_{R}-$ массовый расход дымовых газов, кг/с; $m_{R}-$ масса дымовых газов, кг; $C_{R}-$ удельная теплоемкость дымовых газов, Дж/(кг*К); $T_{R}-$ температура дымовых газов, К; $k_{1}$ - постоянная величина, Вт/K ${ }^{4} ; T_{i n}, T_{\text {out }}$ - температура веществ, которые отдают и получают тепло, К.

Уравнение теплового баланса для конвективного теплообмена.

$$
G_{R} \cdot C_{R 2} \cdot T_{R}-G_{R} \cdot C_{R 2} \cdot T_{\text {out }}=k_{2} G^{0,6}\left(T_{\text {in }}-T_{\text {out }}\right)+m_{2} \cdot C_{R 2} \frac{d T_{m}}{d \tau},
$$

где $G_{R}$ - массовый расход дымовых газов, кг/с; $m_{2}$ - масса дымовых газов, кг; $C_{R 2}-$ удельная теплоемкость дымовых газов по первому участку Дж/(кг*К); $T_{R}, T_{\text {out }}-$ температура дымовых газов за топочной камерой и первым участком, соответственно, К; $T_{i n}$ - температура веществ, отдающих тепло, К; $T_{m}-$ средняя арифметическая температура между температурой дымовых газов топочной камерой и первым участком, К; $k_{2}-$ постоянная величина, $\mathrm{BT} /\left((\kappa г / \mathrm{c})^{0,6} * \mathrm{~K}\right) ; G$ - массовый расход нагреваемого вещества, кг/с.

Уравнение сохранения энергии примет вид: $\frac{d I_{V}}{d \tau}=\frac{d I_{w}}{d \tau}-\frac{d I_{D}}{d \tau}+Q$,

где $I_{V}=V_{W} \rho^{\prime} i^{\prime}+V_{D} \rho^{\prime \prime} i^{\prime \prime}+m_{m} C_{m} T_{m}$ - энтальпия суммарного объема воды, пара и металлических поверхностей труб и самого барабана, Дж; $I_{w}-$ массовая энтальпия потока питательной воды на входе в барабан, Дж; $I_{D}-$ массовая энтальпия пара на выходе из барабана, Дж; $Q$ - суммарный тепловой поток, передаваемый от газов стенкам экранов, 
равный суммарной теплоте, полученной от радиационной и конвективной поверхностей, Вт; $V_{W}, V_{D}-$ объем, который занимает вода и пар в барабане и трубной системе, в соответствии, м ${ }^{3} ; \rho^{\prime}, \rho^{\prime \prime}-$ плотность воды и

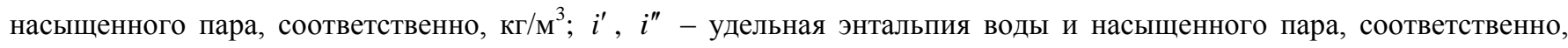
Дж/кг; $m_{m}$ - масса металлических поверхностей труб и барабана, кг; $C_{m}$ - удельная теплоемкость металла, Дж/(кГ*К); $T_{m}$ - температура металла, К.

Объем пароводяной смеси:

$$
\frac{d \Delta P}{d \tau}=\frac{a_{3}-i^{\prime \prime} \cdot a_{2}}{a_{1} \cdot a_{2}-a_{3} \cdot a_{4}} \Delta G_{D}+\frac{a_{2}}{a_{1} \cdot a_{2}-a_{3} \cdot a_{4}}\left(\Delta Q_{1}+\Delta Q_{2}\right),
$$

где $a_{1}=V \cdot \frac{\partial\left(\rho^{\prime \prime} i^{\prime \prime}\right)}{\partial P}+V_{W} \cdot \frac{\partial\left(\rho^{\prime} i^{\prime}-\rho^{\prime \prime} i^{\prime \prime}\right)}{\partial P}+m_{m} C_{m} \cdot \frac{\partial T^{\prime}}{\partial P}-$ постоянный коэффициент, Дж/Па; $a_{2}=\rho^{\prime}-\rho^{\prime \prime}-$ постоянный

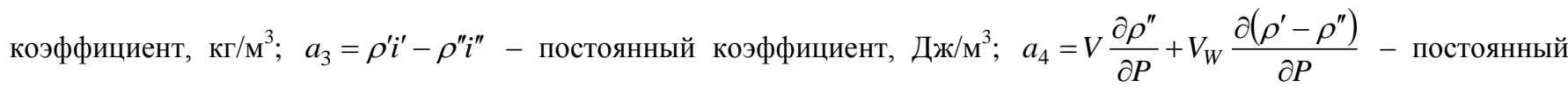
коэффициент, кг/Па; $\Delta P$ - изменение давления в барабане котла; $\Delta G_{D}$ - изменение массового расхода питательной воды, кг/с.

Математическая модель магистрали.

Запишем уравнения сохранения масс, учитывая термодинамические состояния, протекающих в среде, связанные с изменением давления.

$$
G_{\text {in }}-G_{\text {out }}=\frac{G}{p} T \frac{d p}{d \tau}
$$

где $T$ - постоянная времени, с; $G_{i n}, G_{\text {out }}$ - количество вещества на входе и выходе рассматриваемого участка, $\kappa г / \mathrm{c}$.

При этом дифференциальное уравнение для расхода пара в трубопроводе имеет вид:

$$
\begin{aligned}
& \frac{G}{p} T \frac{2 \cdot k_{R} G_{\text {out }} \cdot k_{L} G_{\text {in }}}{k_{R} G_{\text {out }}+k_{L} G_{\text {in }} A_{R}^{2}} \frac{d \Delta G_{\text {out }}}{d \tau}+\Delta G_{\text {out }}=\frac{G}{p} T \frac{2 \cdot k_{R} G_{\text {out }} \cdot k_{L} G_{\text {in }}}{A_{R}\left(k_{R} G_{\text {out }}+k_{L} G_{\text {in }} A_{R}^{2}\right)} \frac{d \Delta A_{R}}{d \tau} \\
& +\frac{k_{R} G_{\text {out }}}{A_{R}\left(k_{R} G_{\text {out }}+k_{L} G_{\text {in }} A_{R}^{2}\right)} \Delta A_{R}+\frac{G}{p} T \frac{k_{L} G_{\text {in }} A_{R}^{2}}{k_{R} G_{\text {out }}+k_{L} G_{\text {in }} A_{R}^{2}} \frac{d \Delta P_{\text {out }}}{d \tau}- \\
& -\frac{A_{R}^{2}}{2\left(k_{R} G_{\text {out }}+k_{L} G_{\text {in }} A_{R}^{2}\right)} \Delta P_{\text {out }}+\frac{A_{R}^{2}}{2\left(k_{R} G_{\text {out }}+k_{L} G_{\text {in }} A_{R}^{2}\right)} \Delta P_{\text {in }}
\end{aligned}
$$

где $G$ - количество вещества на данном участке; $T$ - постоянная времени, с; $G_{i n}, G_{\text {out }}$ - количество вещества на входе и выходе рассматриваемого участка, кг/с; $k_{R}=\frac{\xi_{R}}{2 \rho}$ - коэффициент, определяемый по формуле: $\xi_{R}-$ коэффициент сопротивления регулирующего органа; $k_{L}=\left(\frac{\lambda L}{D_{\text {in }}}+\sum_{i=1}^{n} \xi_{i}\right) \frac{1}{2 \rho S^{2}}-$ коэффициент, определяемый по формуле: $L-$ длина трубопровода, м; $D_{i n}$ - внутренний диаметр трубопровода, м; $\xi$ - коэффициент сопротивления; $\rho$ - плотность газа,

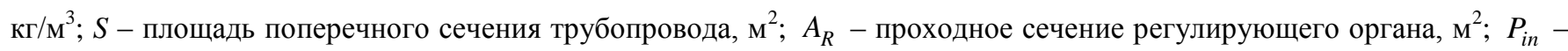
давление в барабане котла, Па; $P_{\text {out }}$ - давление в паропроводе, Па.

5. Метода сжигания углеводородного газа переменного состава.

Для иллюстрации метода определения оптимального отношения топливо/воздух при сжигании углеводородного газа состав которого произвольно меняется во времени, необходимо подготовить данные. Иллюстрацию осуществим на примере случайного изменения во времени составе газа, который находится в газовой магистрали и поступает на сжигание в промышленный барабанный котел. Предположим, что в газовой магистрали случайным образом может находиться метан или этан или этилен или их смесь в следующем соотношении: метан 80 \%, этан 10 \% и этилен $10 \%$.

Обозначим через $V_{\text {ок }}, \dot{V}_{\Gamma}$ - объемный расход окислителя (воздуха) и горючих газов соответственно. Для реализации предложенного алгоритма необходимо следующее техническое обеспечение. Необходима возможность измерений температуры горения газов, объемного расхода воздуха и горючих газов. Кроме того необходима возможность изменения расхода воздуха и горючих газов поступают в котел.

Алгоритм определения оптимального отношения топливо/воздух при сжигании углеводородного газа состав которого произвольно меняется во времени следующий.

Шаг 1. На интервале времени $\Delta \tau$ измеряется температура $T$ и усредняется её значение. На рис. 1 графическая иллюстрация этого измерения показана линией $T=c o n s t$. Измерение температуры осуществляется при фиксированных текущих і-ых значениях $\dot{V}_{\text {окi }}$ и $\dot{V}_{\Gamma i}$. 


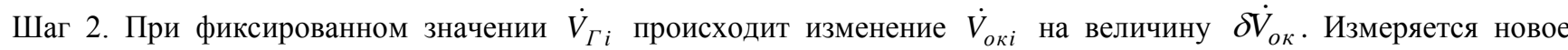
значение температуры $T\left(\dot{V}_{\text {ок }}+\delta \dot{V}_{\text {ок }}\right)$ и определяется характер монотонности на рассматриваемом интервале изменения объёмного расхода воздуха. Если $T\left(\dot{V}_{o \kappa i}\right)>T\left(\dot{V}_{o к i}+\delta \dot{V}_{o \kappa}\right)$, то температура $T$ убывает на этом интервале и в случае $T\left(\dot{V}_{\text {ок }}\right)<T\left(\dot{V}_{\text {ок }}+\delta \dot{V}_{\text {ок }}\right)$ - она растёт см. рис 3. Шаг 2 осуществляется до тех пор, пока дальнейшее $i+1$ изменение $\delta \dot{V}_{o \kappa}$ не приведёт к изменению характера монотонности на противоположный.

Шаг 3. Изменяется знак приращения $\delta \dot{V}_{о \kappa}$ на противоположный и происходит переход к шагу 1.

В таком поисковом режиме в бесконечном временном цикле рассмотрен алгоритм определяет область текущего экстремума. Качественное изменение состава газа в процессе горения приводит к поиску области нового экстремума.

Во-первых, принципиально новая окрестность экстремума значения температуры может находиться как выше, так и ниже текущей зоны и, во-вторых, по значению объемного расхода воздуха $\dot{V}_{\text {ок }}$ - как слева, так и справа от нее.

Рассмотрим на примере сжигания метана, этилена и смеси метана 80\%, этана 10 \% и этилена $10 \%$ с изменением области экстремума, полученной за предложенным алгоритмом см. рис.3.

Предположим, что указанный поисковый алгоритм привел к окрестности точки $\boldsymbol{A}$ кривой (4) и известные текущие $T\left(\dot{V}_{\text {окi }}\right)$ и $\dot{V}_{\text {окi }}$. Допустим, скачкообразно произошла смена качественного состава топлива: на метан кривая (1) или этилен кривая (3). В таком случае при неизменном $\dot{V}_{\text {окi }}$ произойдет изменение температуры $T$ на временном интервале $\Delta \tau$. Если качественный состав будет отвечать этилена, то текущее значение температуры (шаг 1 ) будет находится в окрестности точки $\boldsymbol{B} 1$ на растущей части кривой (3). Если качественный состав будет отвечать метана, то текущее значение температуры (шаг 1) находиться в окрестности точки $\boldsymbol{C 1}$ на нисходящей части кривой (1). Независимо от точки осуществляется приращение $\delta \dot{V}_{o \kappa}$ и определяется характер монотонности текущей кривой. На шаге 2 предложенного алгоритма определяется новая окрестность экстремума точка $\boldsymbol{C}$ - для метана кривая (1), точка $\boldsymbol{B}$ - для этилена кривая (3).

Предложен метод поддержания заданной тепловой нагрузки, основанный на математических моделях вычисления условной формулы газообразного углеводородного топлива и определении динамических характеристик энрегетической установки, что позволило поддерживать максимальный уровень эффективности генерации тепла при использовании углеводородного газа переменного состава за счет постоянного изменения отношения топливо/ воздух с помощью измерений температуры факела и расходов газообразного топлива и окислителя в теплогенерирующей установке.

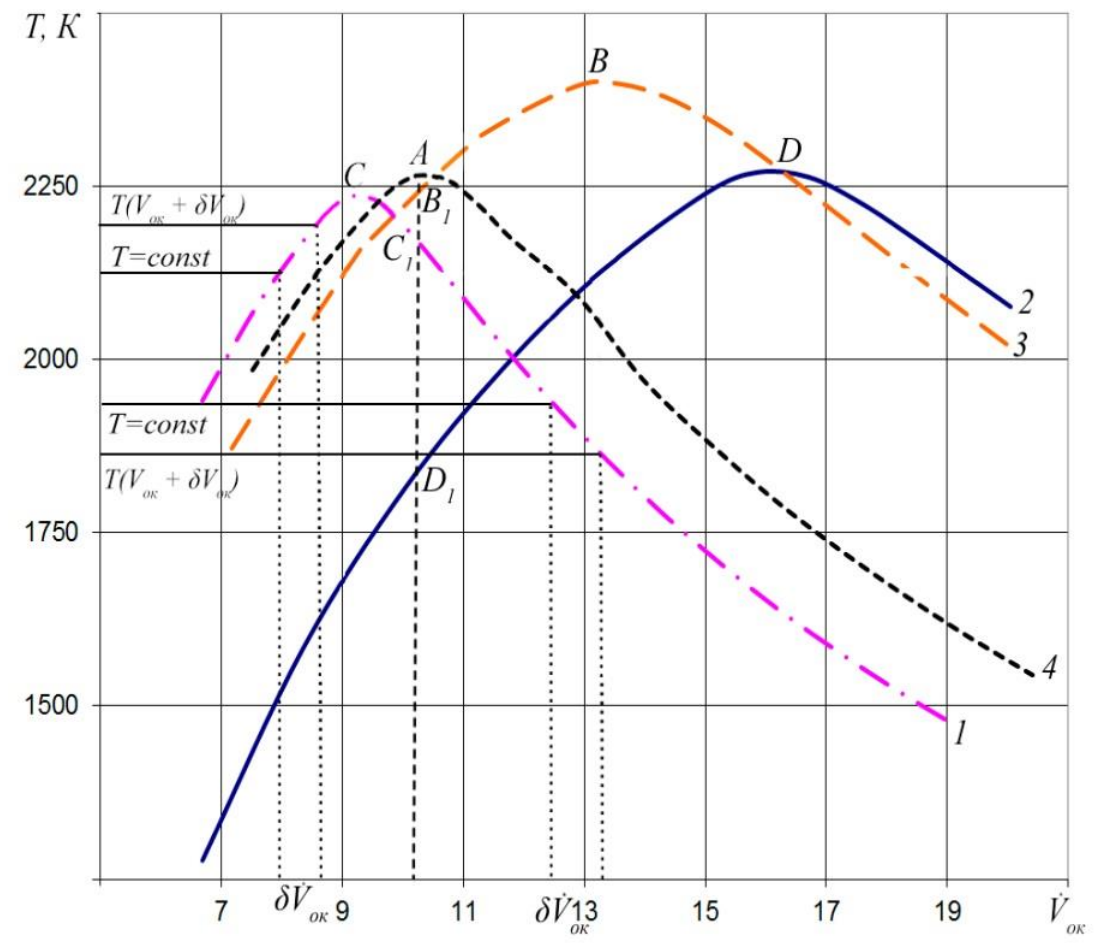

Рис. 1 - Зависимость изменения температуры горения $T$ (метана (1), этана (2), этилена (3), смеси метана 80\%, этана $10 \%$ і этилена $10 \%$ (4)) от объёмногорасхода воздуха $\dot{V}_{o \kappa}$ 


\section{6. Выводы}

1. Для определения условной формулы газообразного углеводородного топлива нашел дальнейшее развитие метод вычисления его условной формулы, основанный на модельных представлениях в соответствии с законами сохранения вещества, Дальтона, химического равновесия по парциальным давлениям. Такой метод позволяет определить количественный состав условной формулы газообразного углеводородного топлива, его энтальпию, состав продуктов сгорания. Предложенная математическая модель отличается от известной модели тем, что в качестве замыкающего соотношения использованы балансы максимальных валентностей окислительных и восстановительных элементов .

2. Для определения динамических характеристик теплоэнергетического оборудования нашла дальнейшее развитие математическая модель, которая состоит из уравнений материального и теплового балансов, законов конвективного и лучистого теплообмена и свойств сжимаемой газообразной среды. Модель отличается от известной тем, что в нее введены зависимости изменения теплотворной способности, и плотности углеводородного газа переменного состава в зависимости от качественного состава. Такая модель позволяет исследовать изменение характеристик парогенерирующего оборудования в установившихся и переходных режимах и определить связь выходных и входных параметров.

3. Для обеспечения заданной тепловой нагрузки предложен метод, базирующийся на математических моделях вычисления условной формулы газообразного углеводородного топлива и определении динамических характеристик парогенерирующей установки. Этот метод дал возможность обесспечивать нахождение оптимального отношения топливо/воздух при использовании углеводородного газа переменного состава за счет постоянного изменения этого отношения с помощью измерений температуры факела в камере сгорания теплогенерирующей установки.

\section{Литература}

[1] Долінський А. Державна цільова програма (проект) модернізації комунальної теплоенергетики на $2010-2014$ роки / А. Долінський, Б. Басок, Є. Базєєв // Вісн. НАН України. - 2009. - № 10. - С. 3-8.

[2] Максимов М.В. Модель и метод определения условной формулы углеводородного топлива при сжигании / М.В. Максимов, А.И. Брунеткин, А.В. Бондаренко // Восточно-европейский журнал передовых технологий. - 2013. Т. 6, № 8 (66). - С. 20-27.

[3] Бондаренко А.В. Исследование высокотемпературного измерительного канала на основе хромельалюминиевых термопар / А.В. Бондаренко, А.И. Брунеткин, А.В. Лысюк // Труды Одесского политехнического университета. - Одесса, 2012. - Вып. 2 (39). - С. 268-272.

[4] Давыдов В.О. Метод расчета температуры горения произвольной смеси газообразного углеводородного топлива при произвольном избытке воздуха / В.О. Давыдов, А.В. Бондаренко // Труды Одесского политехнического университета. - Одесса, 2013. - Вып. 3 (42). - С. 98-102.

[5] Глушко В.П. Термодинамические и теплофизические свойства продуктов сгорания : справ. АН СССР / В.П. Глушко. - ВИНИТИ, 1972. - Т. 1. - 266 с. : ил.

[6] Максимов М.В. Математическая модель сжигания несертифицированных видов топлива / М.В. Максимов, В.Ф. Ложечников, Т.С. Добровольская, А.В. Бондаренко // Восточно-европейский журнал передовых технологий. 2014. - Вып. 2. - С. 47-50.

\section{References}

[1] Dolinskiy, ta in., "Derzhavna tsilova programa (proekt) modernizatsiyi komunalnoyi teploenergetiki na 2010-2014 roki," Vysn. NAN Ukrayini, no. 10 cc. 3-8, 2009;

[2] M. V. Maksimov, "Model i metod opredeleniya uslovnoy formulyi uglevodorodnogo topliva pri szhiganii," Vostochnoevropeyskiy zhurnal peredovyih tehnologiy, vol. 6, no.8(66), pp. 20-27, 2013;

[3] A. V. Bondarenko, i dr., "Issledovanie vyisokotemperaturnogo izmeritelnogo kanala na osnove hromel-alyuminievyih termopar," Trudyi Odesskogo politehnicheskogo universiteta, vyip. 2(39), ss. 268-272, 2012;

[4] V. O. Davyidov i A. V. Bondarenko, "Metod rascheta temperaturyi goreniya proizvolnoy smesi gazoobraznogo uglevodorodnogo topliva pri proizvolnom izbyitke vozduha," Trudyi Odesskogo politehnicheskogo universiteta, vyip. 3(42), ss. 98-102, 2013;

[5] V. P. Glushko, Termodinamicheskie i teplofizicheskie svoystva produktov sgoraniya: cprav. AN SSSR, VINITI.T.1, 1972 ;

[6] M. V. Maksimov, i dr., "Matematicheskaya model szhiganiya nesertifitsirovannyih vidov topliva," Vostochnoevropeyskiy zhurnal peredovyih tehnologiy, vyip. 2, ss. 47-50, 2014. 